\title{
Vestibulotrigeminal pathways in the frog, Rana esculenta
}

\author{
Clara Matesz $^{\mathrm{a}, \mathrm{b}, *}$, Gabriella Kovalecz $^{\mathrm{c}}$, Gábor Veress ${ }^{\mathrm{a}}$, \\ Ádám Deák ${ }^{\mathrm{a}}$, Éva Rácz ${ }^{\mathrm{a}}$, Tímea Bácskai ${ }^{\mathrm{a}}$ \\ a Department of Anatomy, University of Debrecen, Medical and Health Science Center, Debrecen, Hungary \\ ${ }^{\mathrm{b}}$ HAS-UD Neuroscience Research Group MTA-TKI-242, Debrecen, Hungary \\ ${ }^{\mathrm{c}}$ Department of Pediatric Dentistry, University of Debrecen, Medical and Health Science Center, Debrecen, Hungary
}

Received 3 September 2007; received in revised form 18 October 2007; accepted 18 October 2007

\begin{abstract}
The aim of this study was to investigate whether primary vestibular afferent fibers establish direct connections with the motor and sensory trigeminal system in the brainstem of the frog. The experiments were carried out on Rana esculenta. In anaesthetized animals the trigeminal and vestibular nerves were prepared, and their proximal stumps were labeled either with fluorescein binding dextran amine (trigeminal nerve) or tetramethylrhodamine dextran amine (vestibulocochlear nerve). With a confocal laser scanning microscope we could detect close connections between the vestibular fibers and branches of the dorsal dendritic array of the jaw-closing motoneurons, suggestive of monosynaptic contacts. In the other parts of the brainstem, vestibular terminals were detected in the termination areas of the mesencephalic trigeminal nucleus and of the Gasserian (Vth) ganglion and they were probably involved in polysynaptic connections. In agreement with the results obtained in mammalian species, the present findings suggest that the vestibulotrigeminal relationship is quite complex and uses multiple pathways to connect the vestibular apparatus with the motor and sensory nuclei of the trigeminal nerve in the anurans as well.
\end{abstract}

(c) 2007 Published by Elsevier Inc.

Keywords: Neuronal labeling; Confocal laser scanning microscope; Brainstem; Vestibular nuclei; Trigeminal motoneurons

\section{Introduction}

During head movements the maintenance of jaw position and jaw muscle tone is controlled by the activity of the proprioceptors and vestibular receptors. Previous physiological studies on mammalian species revealed that the vestibular stimulus resulted in excitatory influence on the motoneurons of trigeminal nerve innervating the jaw muscles $[3,4,13,14]$. The properties of the vestibular-evoked motor trigeminal response suggested that the activity of vestibular receptors is mediated indirectly to motoneurons by way of polysynaptic pathways. In rats, transneuronal tracing with pseuodorabies virus also provided evidence of a multisynaptic pathway in vestibulotrigeminal connections via neurons of medial and inferior vestibular nuclei [5]. In non-mammalian species, neuronal circuitry between the

Q2 * Corresponding author at: Department of Anatomy, Histology and Embryology, University of Debrecen, Medical and Health Science Center, Nagyerdei krt. 98, H-4012 Debrecen, Hungary. Tel.: +36 52 416 392; fax: +36 52432290.

E-mail address: matesz@ chondron.anat.dote.hu (C. Matesz). vestibular and trigeminal system has not yet been investigated, albeit the previous neuronal labeling experiments are suggestive of the influence of vestibular input to the trigeminal system. With various neuronal labeling techniques, terminals of the afferent vestibular fibers were found in the principal and spinal nucleus of the trigeminal nerve in the frog [7] and in the spinal nucleus of trigeminal nerve of lizard [1] and lamprey [6]. In other experiments, primary afferent trigeminal fibers were followed into the ventral and caudal octaval nuclei of the vestibular nuclear complex of the frog [11]. Extension of dendrites of trigeminal motoneurons into the vestibular nuclear complex may indicate the monosynaptic vestibulotrigeminal connection in the frog $[7,8]$. In this study we have examined the possible anatomical substrates underlying the vestibulotrigeminal neuronal circuitry in the frog.

\section{Materials and methods}

The experiments were carried out on six common water frogs, Rana esculenta in accordance with European Community guidelines and state regulations and with the approval of the University Animal Care Committee. The animals 
were anaesthetized with $0.01 \%$ MS 222 (tricaine methane-sulfonate, SIGMA). The vestibular and trigeminal nerves were prepared from an oropharyngeal approach by an incision of the mucosa on the roof of oral cavity. The cranial cavity was opened by removal of part of the parasphenoidal bone. After incision of the dura mater the trigeminal and vestibular nerves were prepared and transected proximal to the ganglion prooticum commune (the equivalent of the Gasserian ganglion in mammals) and the vestibulocochlear ganglion. Crystals of fluorescein binding dextran amine (FDA, $3000 \mathrm{MW}$, Molecular Probes) were applied to the trigeminal nerve and the tetramethylrhodamine dextran amine (RDA, $3000 \mathrm{MW}$, Molecular Probes) was put on the vestibulocochlear nerve of the same animal. The frogs were kept in a refrigerator for 5 days, reanaesthetized and transcardially perfused with isotonic saline for 2-3 min, and fixed by $4 \%$ paraformaldehyde in $0.1 \mathrm{M}$ phosphate buffer $(\mathrm{pH}$ 7.4). Transverse sections of the brainstem were made with a Vibratome at a thickness of $50 \mu \mathrm{m}$. Images were recorded with an Olympus FV1000 confocal laser scanning microscope $(40 \times$ oil immersion objective, NA $=1.3)$. For the latter analysis we used series of $1 \mu \mathrm{m}$ thick optical slices. We carefully examined the whole XYZ image series. Close appositions were considered if the contact surfaces were at the same focal plane and if there was no discernable gap between the two profiles $[15,17]$. Because of the optical resolution of the objective lens the distance between the surfaces was less than $0.3 \mu \mathrm{m}$. After recording the images we did not use any further image processing.

\section{Results}

Application of FDA to the trigeminal nerve resulted in labeling of sensory fibers and motoneurons similarly to that of the cobalt labeling experiments [8], while RDA was detected in the central terminals of afferent fibers and efferent vestibular neurons in agreement with the earlier results [2,7]. Combination of images displayed overlapping areas of labeled trigeminal and vestibular neurons in different structures of the brainstem. One of them was found in the rostral part of the rhombencephalon at the level of the root of trigeminal nerve. The labeled lateral branches of the dorsal dendritic array of the trigeminal motoneurons extended into the superior (SVN), medial (MVN) and descending (DVN) vestibular nuclei, whereas the vestibular fibers were followed medially and distributed among the dorsal dendritic branches of the trigeminal motoneurons (Fig. 1A). With a confocal laser scanning microscope, we could detect close appositions between the vestibular terminals and the motoneuron dendrites. The closeness of neighboring profiles is
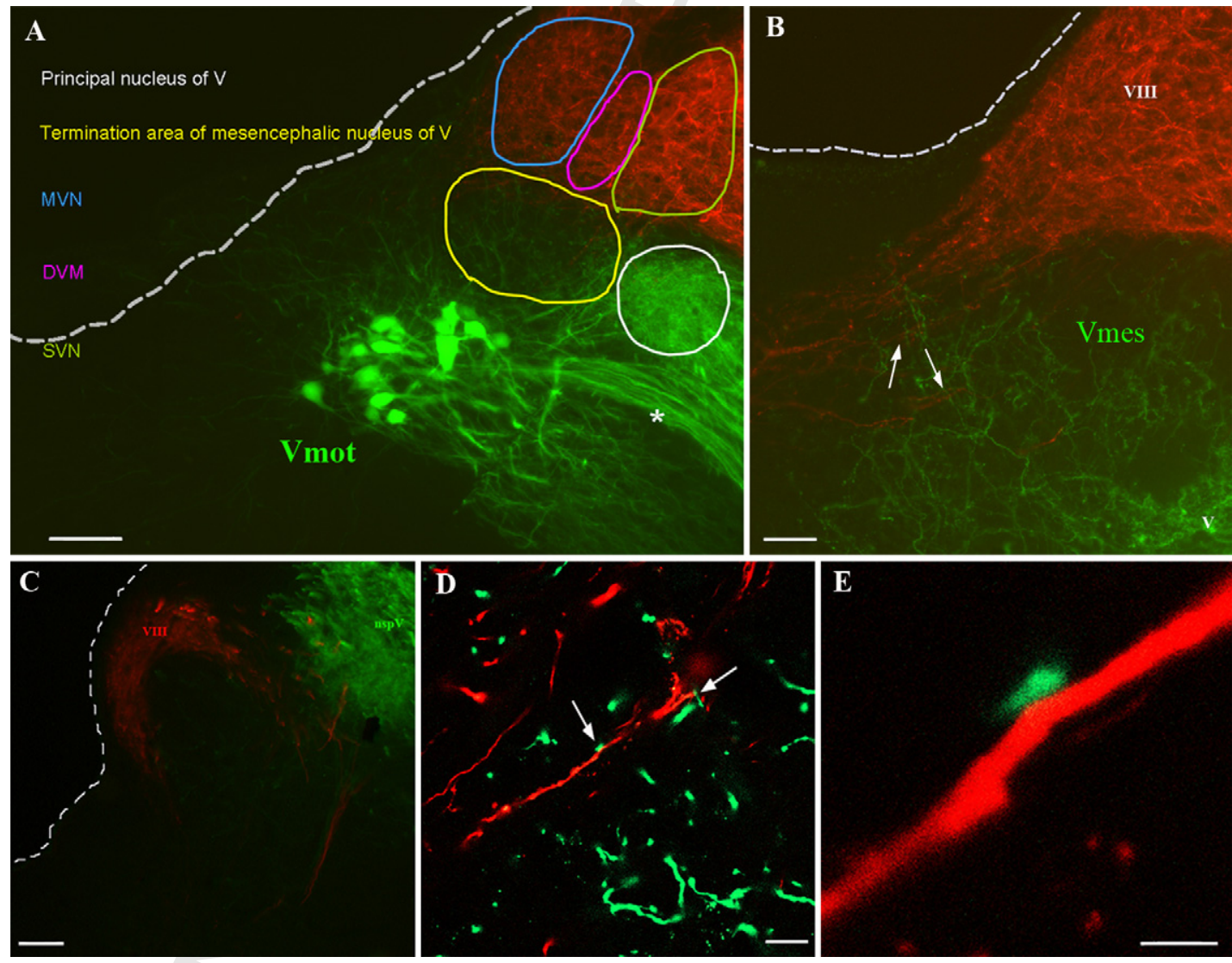

Fig. 1. (A-C) Transverse sections of the brainstem, after labeling of trigeminal nerve with fluorescein binding dextran amine (green) and of the vestibulocochlear nerve with tetramethylrhodamine binding dextran amine (red). (A) At the level of rostral part of the motor trigeminal nucleus the encircled areas with different color show the termination area of the descending limb of the mesencephalic nucleus of trigeminal nerve, as well as the principal nucleus of trigeminal nerve, descending $(\mathrm{DVN})$, medial (MVN) and superior (SVN) vestibular nuclei. The asterisk shows the axons of trigeminal motoneurons. Vmot: motor nucleus of trigeminal nerve. (B) At the level of the rostral part of the ambiguus nucleus the labeled vestibular fibers (arrows) are distributed within the termination area of the descending limb of the mesencephalic nucleus of trigeminal nerve (Vmes). V: Descending tract of trigeminal nerve; VIII: vestibular nuclear complex. (C) At the level of the hypoglossal nucleus vestibular fibers are shown in the spinal nucleus of trigeminal nerve (nspV). (D and E) Confocal photomicrographs, showing the close appositions (arrows) between trigeminal motoneuron dendrites (green) and vestibular fibers (red). Calibration bars $=50 \mu \mathrm{m}(\mathrm{A}-\mathrm{C})$ and $1 \mu \mathrm{m}(\mathrm{D}$ and E).

Please cite this article in press as: C. Matesz, et al., Vestibulotrigeminal pathways in the frog, Rana esculenta, Brain Res. Bull. (2007), doi:10.1016/j.brainresbull.2007.10.049 
suggestive of close membrane appositions without intervening glial or neuronal elements (Fig. 1D and E). Another overlapping areas were detected in the termination fields of the primary sensory neurons of trigeminal nerve throughout the rostrocaudal extent of the vestibular nuclear complex (Fig. 1B and C). The labeled vestibular fibers and terminals were relatively abundant in the termination areas of the descending limb of the mesencephalic trigeminal nucleus and they were sparse in the principal and spinal nucleus of trigeminal nerve, i.e. in the termination areas of the fibers of Gasserian ganglion. The distance between the adjacent boutons of trigeminal, either related to descending limb of the mesencephalic trigeminal nucleus or to the Gasserian ganglion, and vestibular nerve origin indicated that they were separated form each other by an intercalated neuronal or glial element.

\section{Discussion}

Simultaneous labeling of vestibular and trigeminal nerves of the frog revealed a significant overlap of their neuronal elements in the brainstem providing the morphological background for trigeminovestibular pathways. Analysis of confocal images suggested a hitherto unknown monosynaptic connection between motoneurons of the trigeminal nerve and the afferent vestibular fibers of labyrinthine origin. Since the rostral part of the motor trigeminal nucleus contains the motoneurons innervating the jaw-closing muscles $[9,12]$, we assume that the direct labyrinthine input contributes to control the activity of jawclosing muscles during the prey catching behavior.

A large number of vestibular fibers were detected in the termination areas of the primary sensory neurons of trigeminal nerve and they were probably involved in polysynaptic connections. Convergence of sensory modalities involved in the sense of balance has been suggested from the morphological

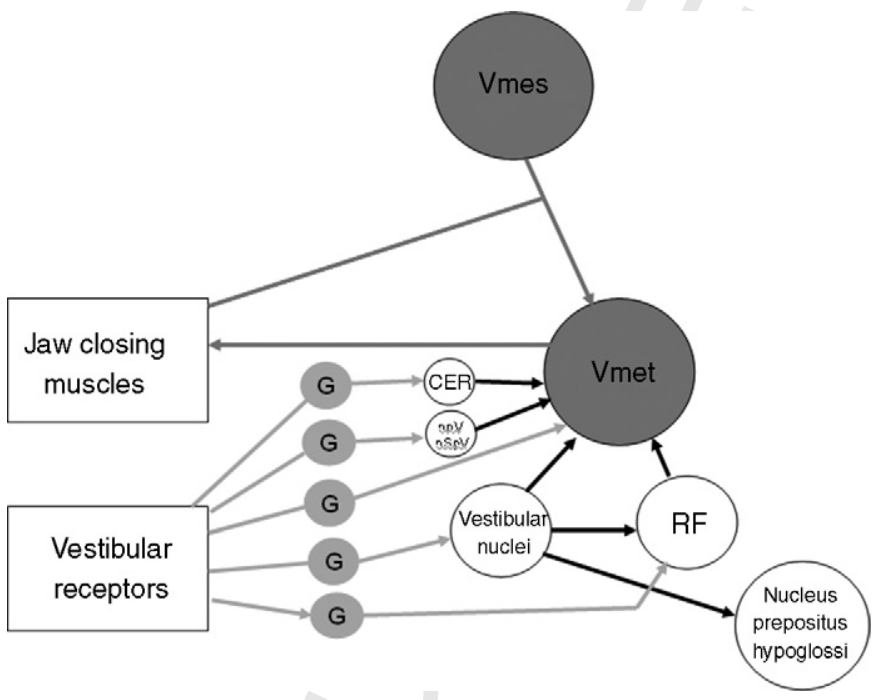

Fig. 2. Summary diagram of the possible vestibulotrigeminal neuronal circuit in the frog. Abbreviations: CER: cerebellum; G: vestibular ganglion; npV: principal nucleus of trigeminal nerve; nspV: spinal nucleus of trigeminal nerve; RF: reticular formation; Vmes: mesencephalic nucleus of trigeminal nerve; Vmot: motor nucleus of trigeminal nerve. and physiological experiments showing a significant overlap of the vestibular and trigeminal input in different parts of the thalamus of the frog $[10,11,16]$. In agreement with the results obtained on mammalian species the present findings suggest that the vestibulotrigeminal relationship is quite complex and uses multiple pathways to connect the vestibular apparatus with the motor and sensory nuclei of trigeminal system in the anurans as well (Fig. 2). The possible monosynaptic and polysynaptic connections between the primary vestibular afferents and the trigeminal system may be one of the underlying mechanisms of a very quick response during the head movements.

\section{Uneited reference}

\section{Acknowledgments}

The authors thank Mrs. B. Szanitter and Miss. Erzsebet Mihaly for skillful technical assistance. This work was supported by MTA-TKI $242 \_$and OTKA K 67641 这

\section{References}

[1] H.A. Barbas-Henry, A.H. Lohman, Primary projections and efferent cells of the VIIIth cranial nerve in the monitor lizard, Varanus exanthematicus, J. Comp. Neurol. 277 (1988) 234-249.

[2] A. Birinyi, H. Straka, C. Matesz, N. Dieringer, Location of dye-coupled second order and of efferent vestibular neurons labeled from individual semicircular canal or otolith organs in the frog., Brain Res. 921 (2001) 44-59.

[3] C. Buisseret-Delmas, C. Compoint, C. Delfini, P. Buisseret, Organisation of reciprocal connections between trigeminal and vestibular nuclei in the rat, J. Comp. Neurol. 409 (1999) 153-168.

[4] M. Diagne, J. Valla, C. Delfini, C. Buisseret-Delmas, P. Buisseret, Trigeminovestibular and trigeminospinal pathways in rats: retrograde tracing compared with glutamic acid decarboxylase and glutamate immunohistochemistry, J. Comp. Neurol. 496 (2006) 759772 .

[5] E. Giaconi, F. Deriu, E. Tolu, B. Cuccurazzu, B.J. Yates, I. Billig, Transneuronal tracing of vestibulo-trigeminal pathways innervating the masseter muscle in the rat, Exp. Brain Res. 171 (2006) 330-339.

[6] H. Koyama, R. Kishida, R.C. Goris, T. Kusunoki, Organization of the primary projections of the lateral line nerves in the lamprey Lampetra japonica, J. Comp. Neurol. 295 (1990) 277-289.

[7] C. Matesz, Central projection of the VIIth cranial nerve in the frog, Neu- Q5 roscience 4 (1979) 20612071

[8] C. Matesz, G. Szekely, The motor column and sensory projections of the branchial cranial nerves in the frog, J. Comp. Neurol. 178 (1978) 157176.

[9] C. Matesz, A. Birinyi, D.S. Kothalawala, G. Székely, Investigation of the dendritic geometry of brainstem motoneurons with different functions using multivariant multistatistical techniques in the frog, Neuroscience 65 (1995) 1129-1144.

[10] C. Matesz, A. Kulik, T. Bácskai, Ascending and descending projections of the lateral vestibular nucleus in the frog Rana esculenta, J. Comp. Neurol. 444 (2002) 115-128.

[1z] M. Muňoz, A. Muňoz, O. Marin, A. Gonzalez, Primary afferents and second order projections of the trigeminal system in a frog (Rana ridibunda), Eur. J. Morphol. 32 (1994) 288-292.

[13] G. Szekely, C. Matesz, The efferent system of cranial nerve nuclei: a comparative neuromorphological study, Adv. Anat. Embryol. Cell Biol. 128 (1993) 1-92.
140

141

168 
[14] E. Tolu, M. Pugliatti, The vestibular system modulates masseter muscle activity, J. Vestib. Res. 3 (1993) 163-171.

[15] J. Valla, C. Delfini, M. Diagne, G. Pinganaud, P. Buisseret, C. BuisseretDelmas, Vestibulotrigeminal and vestibulospinal projections in rats: retrograde tracing coupled to glutamic acid decarboxylase immunoreactivity, Neurosci. Lett. 340 (2003) 225-228.

[16] T. van Haeften, F.G. Wouterlood, Neuroanatomical tracing at high resolution, J. Neurosci. Meth. 103 (2000) 107-116.
[17] G. Westhoff, G. Roth, H. Straka, Topographic representation of vestibular and somatosensory signals in the anuran thalamus, Neuroscience 124 (2004) 669-683.

[18] F.G. Wouterlood, T. Bocker, M.P. Witter, Synaptic contacts between identified neurons visualized in the confocal laser scanning microscope, neuroanatomical tracing combined with immunofluorescence detection of post-synaptic density proteins and target neuron-markers, J. Neurosci. Methods 128 (2003) 129-142.

Please cite this article in press as: C. Matesz, et al., Vestibulotrigeminal pathways in the frog, Rana esculenta, Brain Res. Bull. (2007), doi:10.1016/j.brainresbull.2007.10.049 